\title{
A miniprocessor PDP8/e-based system for investigations of on-line language processing: Automated program for psycholinguistic experiments (APPLE)
}

\author{
WILLIAM ONIFER, MAX HIRSHKOWITZ, and DAVID SWINNEY \\ Tufts University, Medford, Massachusetts 02155
}

\begin{abstract}
An on-line reaction time program designed for psycholinguistics research implemented on a DEC PDP8/e minicomputer is described. Current applications include: phoneme monitor, click detection, lexical decision, and sentence verification experiments. This assembly language program (PAL8) displays alphanumeric texts on remoted oscilloscopes and records decisiondependent response times with 1-msec accuracy. The system allows for simultaneous testing of three subjects. It operates either according to internally generated timing or in synchrony with audio tape. Individuals with little or no computer background can develop and run experiments without difficulty.
\end{abstract}

Recent research in psycholinguistics has increasingly relied upon measures of processing that are on-line, in the sense that they are taken while the language operation of interest is occurring. On-line measures are necessary because they are not distorted by memory encoding effects and they allow investigation of effects that have limited time durations (e.g., derivation of word meaning during ongoing speech comprehension). Most aspects of language perception appear to be such rapid and complex processes that evidence of their processing sequences and stages quickly fades or is obscured. On-line decision-dependent reaction time measures such as the phoneme monitor task, click location task, lexical decision task, and sentence verification task have been successfully employed in investigations of numerous aspects of linguistic processes. Word encoding and recoding (Meyer, Schvaneveldt, \& Ruddy, 1975; Rubenstein, Lewis, \& Rubenstein, 1971), context effects on the perception of ambiguous words (Swinney, Note 1), and processing effects of syntactic variables (Abrams \& Bever, 1969) have been studied.

In order to facilitate the development and execution of research utilizing response time as a dependent measure, a miniprocessor PDP8/e-based system has been developed. The system is designed to allow individuals with little or no computer background to implement research using any of the previously mentioned experimental paradigms.

\section{PROGRAM DESCRIPTION}

This system (APPLE) provides: (1) visual presentation of alphanumeric strings on standard $X-Y$ driven oscilloscopes; (2) the simultaneous recording of responses and reaction times from three subjects; (3) internal or external timing for stimulus presentation and interstimulus interval (ISI); (4) random or nonrandom sequence of stimulus presentation; and (5) hard-copy output and/or mass storage of experimental data.

Each of the items for visual display is limited to a single line with a maximum of 42 characters that appear in uppercase on a 6 by 5 (height by width) point matrix. This program allows for up to 8,000 characters with a maximum of 650 separate displays. Items can be displayed for a set duration ranging from .01 to $4.00 \mathrm{sec}$. The response period begins at the onset of stimulus display. Length of the response period is variable with a maximum of $4.00 \mathrm{sec}$. Reaction times (in milliseconds) and subject responses (recognition or decision responses provided by buttonpress) are recorded. Stimulus presentations are initiated either by internally generated timing or by an externally provided digital input signal. The internal timing allows for random or fixed interstimulus intervals that range from .01 to $20.48 \mathrm{sec}$. External timing is customarily provided by a tapeactivated relay that synchronizes the visual display with auditorially presented material. The order of stimulus presentation can either be fixed (in order of entry) or random (without replacement).

At the conclusion of an experimental run, a hardcopy output of the presented items, along with the subjects' decisions and response latencies, is generated which corresponds to the original order of item entry.

\section{HARDWARE REQUIREMENT}

At present APPLE is implemented on a PDP8/e; however, it should be easily adaptable to any OS8-based system. The required hardware includes: $12 \mathrm{~K}$ of memory, a programmable clock, two digital-to-analog converters, a 12-bit digital input device, a terminal, 
and a mass storage device. Additionally, for each subject, one X-Y drivable oscilloscope, two response buttons, and stereo headphones are needed. For experiments in which item presentation is concurrent with auditorially presented information, a tape recorder equipped with a tape-activated relay is necessary. In order to maintain stimulus display quality on remotely placed oscilloscopes, the display signals are amplified with $15-\mathrm{V}$ dc amplifiers.

\section{PROGRAM AND OPERATION}

The logic flow diagram (Figure 1) depicts the basic sequence of program operation. To utilize this program for research, the user must first create an ASCII file containing the text strings to be displayed and a text address directory (pointers). This is accomplished by inserting the desired text strings into an existent master file that contains all necessary auxiliary coding. The text file must then be assembled (with PAL8 or other OS8 assembler) to generate a binary file of packed ASCII characters and pointers. The binary file is then combined with APPLE to produce an executable program.

Upon execution, the system initiates a dialogue with the user to determine the parameters and options required for the run. A digital input initiates item presentation. Following the start of an experiment, APPLE controls all aspects of stimulus presentation and data collection. Program operation can be temporarily suspended and continued by providing digital inputs. At the conclusion of a run, a hard-copy output of the data is generated, and the data may be stored for off-line analysis. Auxiliary software is presently used to pass (via MODEM) data to a DEC 10 system for statistical evaluation by ALICE (Walker, Bauer, \& Grubin, 1976) or other analysis packages.

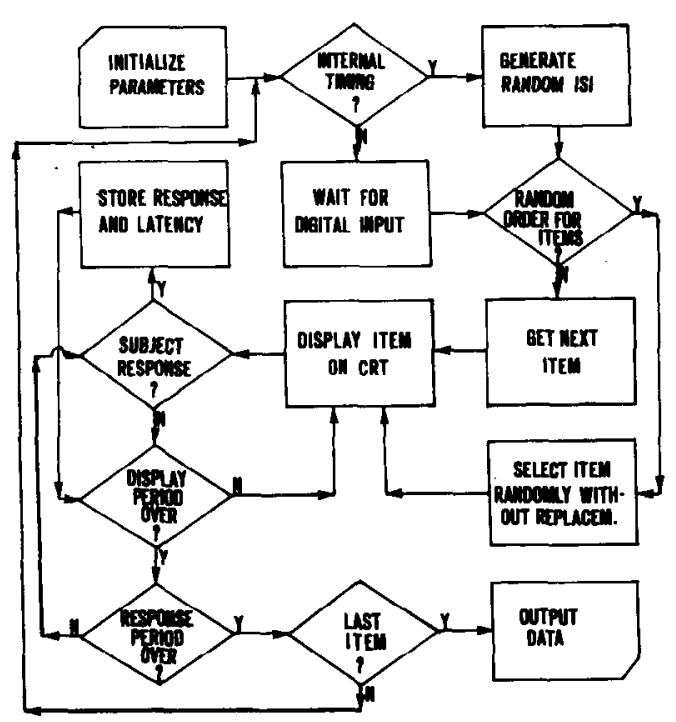

Figure 1. Logic flow diagram: the basic flow of program operation.

\section{PROGRAM APPLICATION}

Current applications of this system include a variety of psycholinguistic research projects. Lexical decision task (i.e., word verification) experiments are easily conducted with APPLE. This paradigm has been utilized to investigate the structure and organization of semantic memory. The tape-synchronized timing feature of this system has been used to investigate language processing during ongoing sentence perception; for example, the effects of semantic and syntactic context on the processing of ambiguous lexical items (Swinney, Note 1; Prather \& Swinney, Note 2).

\section{SUMMARY}

APPLE provides researchers with a powerful and adaptable tool for investigating psycholinguistic phenomena. It allows easy experiment implementation and automatic simultaneous data collection from several subjects. The use of a lab miniprocessor and conventional computer peripherals eliminates the need for elaborate special-purpose hardware. Moreover, the development of system software provides the versatility and flexibility necessary for experimental research in a small laboratory. System development on a general-purpose lab computer allows individuals in a variety of different research areas to conduct sophisticated experiments economically and efficiently. Finally, while software development is possible and often easier in higher level languages (e.g., FORTRAN), system development at the machine language level alleviates limitations and restrictions imposed by peripheral $\mathrm{l} / \mathrm{O}$ handling operation speed.

\section{REFERENCE NOTES}

1. Swinney, D. Does context direct lexical access? Paper presented at the annual meeting of the Midwestern Psychological Association, Chicago, 1976.

2. Prather, P., \& Swinney, D. Some effects of syntactic context upon lexical access. Paper presented at the annual meeting of the American Psychological Association, San Francisco, August 1977.

\section{REFERENCES}

Abrams, K., \& Bever, T. G. Syntactic structure modifies structure during speech perception and recognition. Quarterly Joumal of Experimental Psychology, 1969, 21, 280-290.

MeYer, D. E., Schvaneveldt, R. W., \& Ruddy, M. G. Loci of contextual effects on visual word recognition. In P. M. A. Rabbit \& S. Dornic (Eds.), Attention and Performance V. London: Academic Press, 1975. Pp. 98-118.

Rubenstein, H., Lewis, S. S., \& Rubenstein, M. A. Evidence for phonemic recoding in visual word recognition. Journal of Verbal Learning and Verbal Behavior, 1971, 10, 645-657.

Walker, E., Bauer, J. A., \& Grubin, M. L. Alice: A system for manipulating and analyzing multidimensional data. Behavior Research Methods \& Instrumentation, 1976, 8, 347-351. 